\title{
Fulminant necrotizing streptococcal myositis with dramatic outcome - a rare case report
}

Tudor Morar ${ }^{1}$, Radu Pirlog ${ }^{1,2}$, Sonia Vlaicu ${ }^{3}$, Vasile Bintintan ${ }^{4}$, Doinita Crisan $^{1}$

1) Department of Morphological Sciences, Iuliu Hațieganu University of Medicine and Pharmacy, ClujNapoca, Romania

2) Research Center for Functional Genomics, Biomedicine and Translational Medicine, Iuliu Hatieganu University of Medicine and Pharmacy, Cluj-Napoca, Romania

3) Department of Internal Medicine, Iuliu Hatieganu University of Medicine and Pharmacy, Cluj-Napoca, Romania

4) $1^{\text {st }}$ Clinic of Surgery, Iuliu Hațieganu University of Medicine and Pharmacy, Cluj-Napoca, Romania

DOI: $10.15386 / \mathrm{mpr}-1866$

Manuscript received: 24.08 .2020

Received in revised form: 15.11.2020

Accepted: 29.11.2020

Address for correspondence:

Pirlog.radu@umfcluj.ro

This work is licensed under a Creative Commons Attribution-NonCommercialNoDerivatives 4.0 International License

\begin{abstract}
Necrotizing myositis represents a rare, aggressive form of bacterial-induced soft tissue necrotizing infection. We present a fulminant case of a 44-year-old patient with a necrotizing soft tissue infection and a history of rheumatoid arthritis transferred to our service, Cluj-Napoca Emergency County Hospital, from a local hospital where he had been admitted two days before with chills and light-headedness after an accidental minor blunt trauma in the right thigh region. After admission to our hospital and first assessment, broad spectrum antibiotherapy was started with Meropenem, Vancomycin and Metronidazole along with surgical debridement. The evolution was fulminant with rapid development of multiple organ dysfunction syndrome, therefore he was transferred to the intensive care unit, intubated, and started the volemic resuscitation and vasopressor therapy. The blood culture was positive for group A beta-hemolytic streptococcus (GAS) and high dose Penicillin $\mathrm{G}$ was added to the therapeutic scheme. Despite all efforts, the patient developed disseminated intravascular coagulation syndrome and died in the next hours. The clinical picture together with the findings from the autopsy were suggestive for a streptococcal toxic shock syndrome developed as a complication of GAS induced necrotizing myositis.
\end{abstract}

Keywords: necrotizing myositis, soft tissue infection, immune suppression, streptococcal toxic shock syndrome

\section{Introduction}

Necrotizing myositis represents a fulminant, life-threatening form of necrotizing soft tissue infection (NSTI) that involves fascia, subcutaneous tissue and muscle. It is typically associated with the presence of group A beta-hemolytic streptococcus (GAS, Streptococcus pyogenes), but also other 'flesh-eating bacteria' such as Peptostreptocous spp., Fusobacterium spp., Bacteroides spp., and Enterobacterales [1]. Clinically it is characterized by fast, massive tissue destruction and signs of systemic toxicity. Bacteria cross the skin barrier through small entry points caused by scratches and punctures that could occur even without noticing during daily activities. Thus, the most commonly affected parts are usually the lower limbs.
The initial signs are often non-specific with minimum inflammatory signs, hence a high level of clinical suspicion must be in place to diagnose early stages of disease and to boost survival chances. In general, patients have predisposing conditions like diabetes mellitus or various forms of immunodeficiencies, acquired or iatrogenic. The most common clinical presentation of these patients are septicemia, cellulitis or abscess. The standard of care is emergency surgical intervention with massive debridement of all necrotic tissues and broad spectrum antibiotherapy. Mortality rates are high, currently even with the most recent advances in medicine mortality is around $20 \%$ and is touching $100 \%$ in the absence of immediate medical intervention [2,3]. 


\section{Case report}

A 44 years old Caucasian male with a previous history of rheumatoid arthritis for which he was receiving immunosuppressive treatment with Methotrexate and Tocilizumab presented to a local rheumatology ward with chills and light-headedness. Two days prior to hospital presentation the patient admitted having an accidental blunt trauma in the right thigh region while working in the yard. There were apparently no skin breaches, or subcutaneous crepitus at the trauma site. Soon the general state altered with high fever, local muscle pain and right lower limb edema accompanied by blisters formation and the development of a bluish-black coloration in the trauma cutaneous corresponding area. Therefore, the patient was urgently transferred from a local hospital to our facility at the ClujNapoca Emergency County Hospital. The initial assessment suggested a necrotizing soft tissue infection which lead to the urgent initiation of the mixed pharmacological and surgical treatment with broad spectrum antibiotherapy with Meropenem, Vancomycin and Metronidazole was started along with three decompressive fasciotomies. During the surgical assessment the superficial muscle fascia seemed viable, there were no fetid discharges, but the right and medial vastus femoral muscles had incipient necrotic areas for which debridement was performed. The patient became rapidly obnubilated, dyspneic, with blood gas analysis showing hypoxemia, marked metabolic acidosis with high lactate levels, which required immediate orotracheal intubation. The patient started to develop multiple organ dysfunction syndrome, he became hypotensive $(\mathrm{BP}=60 / 34 \mathrm{mmHg})$ and tachycardic (160 beats/minute). He was transferred to the intensive care unit where aggressive volemic resuscitation was started along with vasopressor therapy. The patient was febrile $\left(\mathrm{t}=38.3^{\circ} \mathrm{C}\right)$, severely pancytopenic $(\mathrm{L}=1530$ / $\left.\mu \mathrm{L}, \quad \mathrm{RBC}=1.8 \times 10^{6} / \mu \mathrm{L}, \mathrm{Tr}=15000 / \mu \mathrm{L}\right)$, hypoproteinemic (total proteins $=2.7 \mathrm{~g} / \mathrm{dL}$ ), presenting low serum fibrinogen, spontaneously prolonged coagulation $(\mathrm{INR}=3)$, marked renal dysfunction (creatinine $4.25 \mathrm{mg} / \mathrm{dL}, \mathrm{BUN}=120 \mathrm{mg}$ / $\mathrm{dL})$ and increased markers of inflammation $(\mathrm{CRP}=13 \mathrm{mg} /$ $\mathrm{dL})$. Severe rhabdomyolysis was present $(\mathrm{CK}=22178 \mathrm{U} / \mathrm{L}$, almost 130 times above normal value), along with increasing levels of transaminases (ALAT $=1715 \mathrm{U} / \mathrm{L}, \mathrm{ASAT}=6381$ $\mathrm{U} / \mathrm{L}$ ), serum bilirubin (total bilirubin $4,58 \mathrm{mg} / \mathrm{dL}$ ) and lactate dehydrogenase $(\mathrm{LDH}=3969 \mathrm{U} / \mathrm{L})$. A constant oozing hemorrhage could be noted at the site of the surgical wound. Blood transfusions comprising erythrocytes and platelets concentrates, fresh frozen plasma, cryoprecipitate, as well as administration of prothrombin complex and activated factor VII were undertaken considering the presence of intravascular disseminated coagulation syndrome with profound bleeding. Intermittent hemodiafiltration was instituted. Both blood and tissue cultures grew GAS, so high dose Penicillin G was added to the therapeutic plan. Local surgical hemostasis was attempted in the first instance, but soon afterwards the patient returned to the operating theatre given the rapid extension of tissue necrosis. Extensive debridement comprising skin, subcutaneous fatty tissues, superficial fascia, thigh muscles leaving femoral periosteum exposed, scrotum and perineal tissues was undertaken. Despite vigorous medical efforts the patient's death occurred less than 48 hours after the arrival in our facility. Autopsy was performed and findings included multiple elevated vesicular lesions on the skin of arms and left thigh (Figure 1).

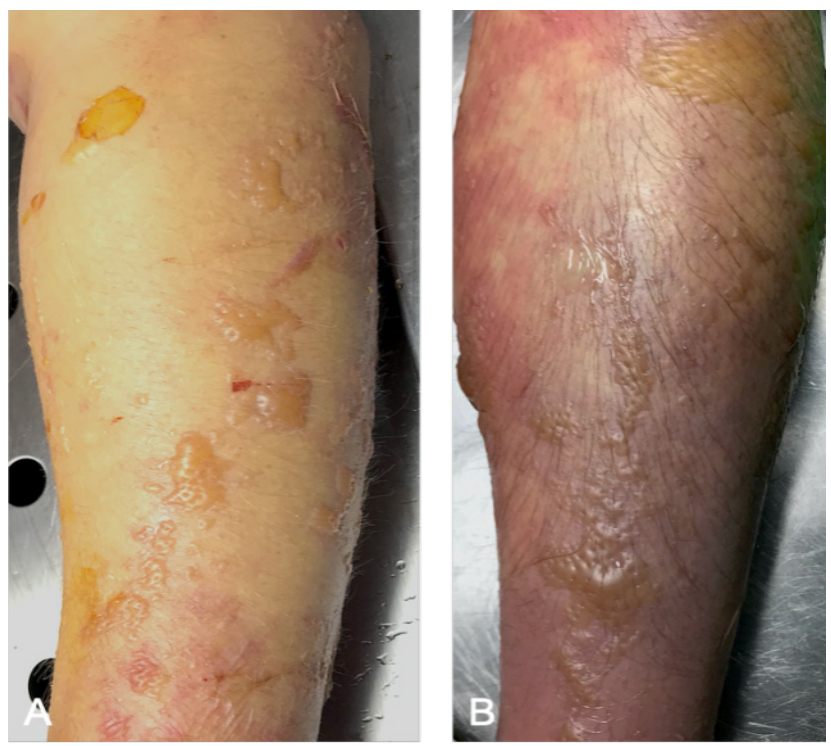

Figure 1. A - left forearm, B - Left leg. Superficial skin yellow vesicles filled with liquid.

The scrotum was removed and the testicles exposed due to surgical intervention. Inflammatory lymphadenopathy was noted in the femoral triangle (Figure 2).

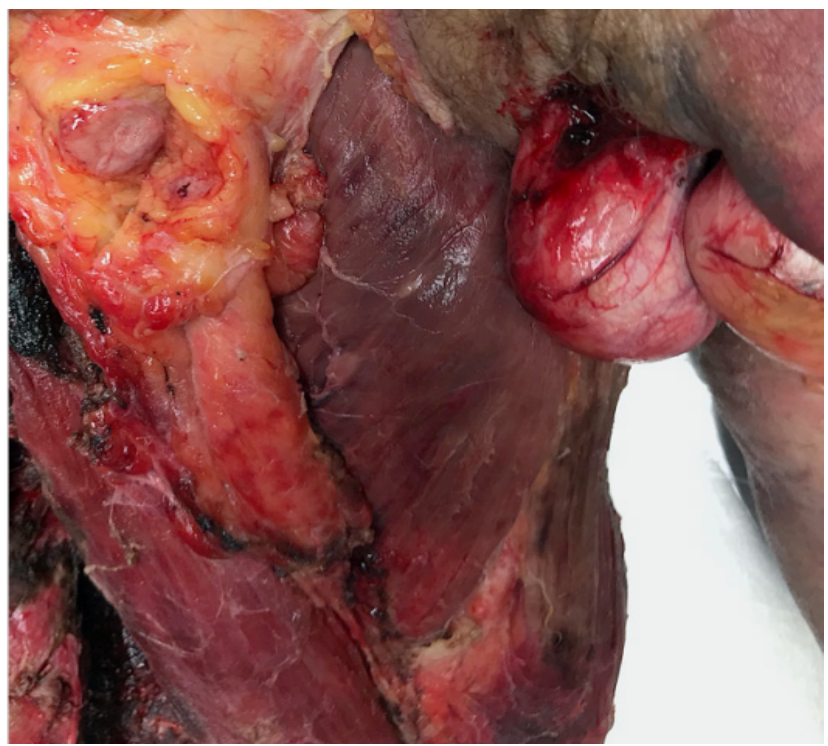

Figure 2. An inflammatory inguinal lymphadenopathy can be seen in the upper left, along with the exposed testicles after massive debridement. 

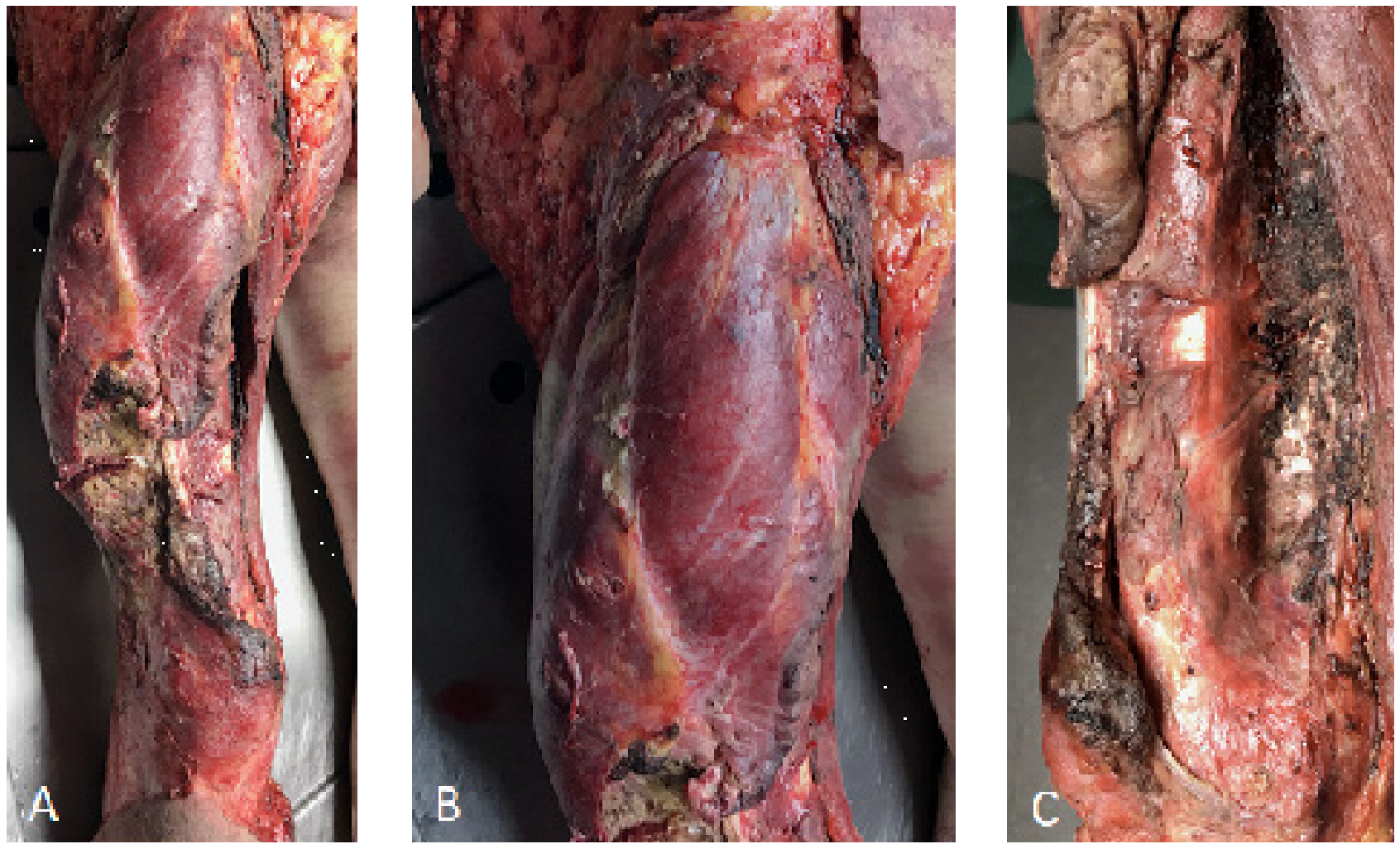

Figure 3. A - Right inferior leg after surgery debridement of the affected skin, fascia and muscles. B - Lateral view. C - Medial view.
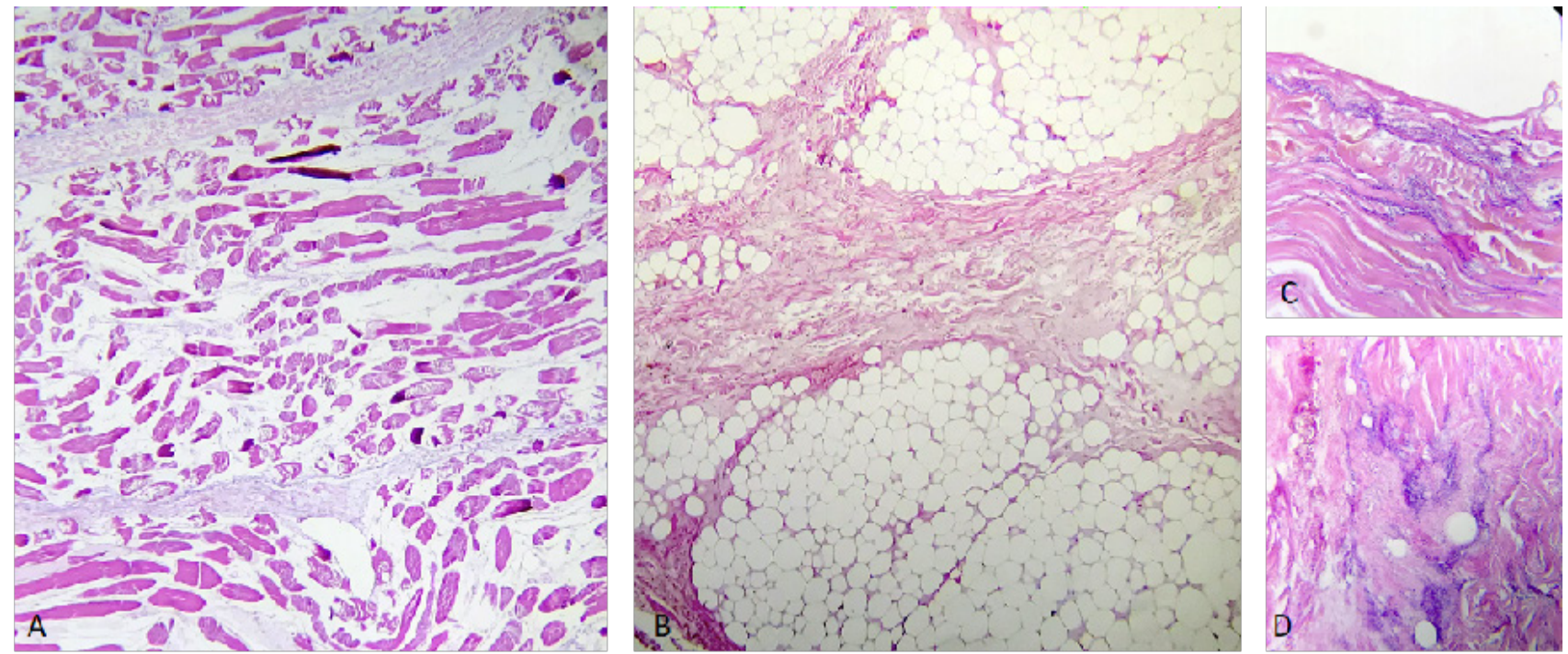

Figure 4. All the images are stained using hematoxylin-eosin. A- muscular and fascial necrosis. B - fat necrosis and fascial necrosis. C \& D - bacterial colonies, gas vesicles and fascial necrosis.

The right hip and thigh were severely affected by the progressive disease and due to extensive debridement of the skin and subjacent tissues the muscles were exposed (Figure 3).
Histological examination of the surgical specimens yielded the presence of extensive necrosis including hypodermic fat, fascia and muscle tissue, with widespread vascular thrombosis, bacterial colonies and gas bubbles distorting normal tissue architecture (Figure 4). 


\section{Discussion}

Necrotizing myositis represents a life-threatening condition, requiring a high level of suspicion in order to perform a quick diagnosis, which is the sine qua non condition to improving prognosis [4]. The main etiologic agent described in literature is GAS (Streptoccus pyogenes), although other pathogens like Clostridium, Staphylococcus, Vibrio, Aeromonas and Pasteurella have been identified $[5,6]$. Major risk factors associated with invasiveness are minor trauma, use of nonsteroidal antiinflammatory drugs, recent surgery, obesity, poor socioeconomic status, malignancy and immunosuppression [7]. In the particular case of our patient both minor local trauma and pharmacological immunosuppression could be identified as known risk factors for NSTI. Patient treatment consisted in a combination of Methotrexate and Tocilizumab which have been previously individually described as risk factors for NSTI $[8,9]$. In our case we speculate that this combination of two immune system suppressants, could be the favoring factor for the fulminant evolution leading to death. Pathogenesis is incompletely understood and implies complex interactions between the human host defense mechanisms and specific bacterial virulence factors. Of those we mention the cell surface $M$ protein and streptococcal exotoxins $[10,11]$. The increasing prevalence of M1 and M3 subtypes seems to be responsible for the increasing number of severe streptococcal invasive infections in the last years $[12,13]$. Also, these strains have been more often associated with the streptococcal toxic shock syndrome (STSS). STSS represents a feared complication of GAS invasive infections, occurring in up to one third of cases [14]. It is defined by hypotension and multiple organ failure, with renal impairment, coagulopathy, liver and respiratory failure [7]. The streptococcal exotoxins are the center of its pathogenesis by inducing the release of inflammatory cytokines leading to tissue damage and increased capillary leak. Treatment resides in fluid management, respiratory ventilation, vasopressor support, renal replacement therapy, along with antibiotics, draining off the source of infection and adjunctive therapies like intravenous immunoglobulins. Despite energetic efforts, STSS is characterized by extremely high mortality rates.

Laboratory findings are nonspecific and positive diagnosis relies on quick, thorough surgical examination corelated with microbiological tissue or secretions result and the histopathological exam of the necrotic debridement specimens [15]. Imagistic approaches are also of little value to diagnosis as many findings are once again nonspecific [16]. Therapeutic pillars consist in aggressive surgical debridement until reaching healthy tissue, empiric broad spectrum antibiotherapy that is later tailored to culture results and hemodynamic resuscitation. Multiple interventions might be needed to control the spread of infection, which could lead to limb amputation as a last solution that should not be overlooked. Antibiotherapy should cover the spectrum of causative microorganisms: Streptococcus pyogenes, Staphylococcus aureus, Methicillin-resistant Staphylococcus aureus, Gram-negative aerobes and anaerobes [17]. Two adjuvant therapies currently used are: hyperbaric oxygen therapy (HBOT) and intravenous immunoglobulins (IVIG). HBOT aims to improve tissue oxygenation, thus preventing an anaerobiotic environment, but has failed to prove a mortality benefit [18]. IVIGs are favored in the setting of NSTIs associated with toxic shock syndrome in order to neutralize bacterial exotoxins and limit systemic inflammation. If the patient survives, plastic surgery with skin and grafts is needed in an attempt to restore functionality and esthetics.

\section{Conclusion}

We described a fulminant case of necrotizing myositis in a young, but pharmacologically immunosuppressed underlining the extreme aggression of soft tissue streptococcal infections. Integrating the clinical, paraclinical and autopsy findings we conclude that this case was a GAS induced necrotizing myositis complicated with STSS which was responsible for the systemic manifestations. This entity should be considered by surgeons, dermatologists and emergency medicine professionals especially in patients undergoing immunosuppressive treatment. Furthermore, double immunosuppression, as seen in this case, should be evaluated with care as it may pose patients to an additional risk. Clinicians must be aware of this entity and carefully screen potential patients for it to ensure best outcomes for those affected. In these diseases time is both flesh and life.

\section{References}

1. Wong $\mathrm{CH}$, Chang HC, Pasupathy S, Khin LW, Tan JL, Low CO. Necrotizing fasciitis: clinical presentation, microbiology, and determinants of mortality. J Bone Joint Surg Am. 2003;85:1454-1460.

2. Al-Qurayshi Z, Nichols RL, Killackey MT, Kandil E. Mortality Risk in Necrotizing Fasciitis: National Prevalence, Trend, and Burden. Surg Infect (Larchmt). 2020;21:840-852.

3. Karnuta J, Featherall J, Lawrenz J, Gordon J, Golubovsky $\mathrm{J}$, Thomas J, et al. What Demographic and Clinical Factors Are Associated with In-hospital Mortality in Patients with Necrotizing Fasciitis? Clin Orthop Relat Res. 2020;478:17701779 .

4. Adair A, MacDonald L, Alasadi A, Holdsworth RJ. Streptococcal myositis a surgical emergency. Surgeon. 2009;7:128.

5. Frazee BW, Fee C, Lynn J, Wang R, Bostrom A, Hargis C, et al. Community-acquired necrotizing soft tissue infections: a review of 122 cases presenting to a single emergency department over 12 years. J Emerg Med. 2008;34:139-146.

6. Izumi M, Hiraiwa T, Tomioka H, Komura M, Hayashi Y. Fatal necrotizing myositis from fulminant Streptococcus dysgalactiae subspecies equisimilis (SDSE) infection: A case report of autopsy images. J Gen Fam Med. 2018;19:50-52. 
7. Schmitz M, Roux X, Huttner B, Pugin J. Streptococcal toxic shock syndrome in the intensive care unit. Ann Intensive Care. 2018;8:88.

8. Sullivan C, Sullivan P, Eadie P, Veale D. Necrotizing fasciitis in patients on methotrexate therapy. J Clin Rheumatol. 2013;19:289-291.

9. Manabe S, Yanagi H, Ozawa H, Takagi A. Necrotising soft tissue infection without systemic toxicity in a patient with rheumatoid arthritis treated with tocilizumab. BMJ Case Rep. 2017;2017:bcr2017222826.

10. Frost HR, Sanderson-Smith M, Walker M, Botteaux A, Smeesters PR. Group A streptococcal M-like proteins: From pathogenesis to vaccine potential. FEMS Microbiol Rev. 2018;42:193-204.

11. Barsumian EL, Schlievert PM, Watson DW. Nonspecific and specific immunological mitogenicity by group A streptococcal pyrogenic exotoxins. Infect Immun. 1978;22:681-688.

12. Tyrrell GJ, Lovgren M, Forwick B, Hoe NP, Musser JM, Talbot JA. M types of group a streptococcal isolates submitted to the National Centre for Streptococcus (Canada) from 1993 to 1999. J Clin Microbiol. 2002;40:4466-4471.
13. Moses AE, Goldberg S, Korenman Z, Ravins M, Hanski E, Shapiro M. Invasive group a streptococcal infections, Israel. Emerg Infect Dis. 2002;8:421-426.

14. Stevens DL, Tanner MH, Winship J, Swarts R, Ries KM, Schlievert PM, et al. Severe group A streptococcal infections associated with a toxic shock-like syndrome and scarlet fever toxin A. N Engl J Med. 1989;321:1-7.

15. Neeki MM, Dong F, Au C, Toy J, Khoshab N, Lee C, et al. Evaluating the Laboratory Risk Indicator to Differentiate Cellulitis from Necrotizing Fasciitis in the Emergency Department. West J Emerg Med. 2017;18:684-689.

16. Tang WM, Wong JW, Wong LL, Leong JC. Streptococcal necrotizing myositis: the role of magnetic resonance imaging. A case report. J Bone Joint Surg Am. 2001;83:1723-1726.

17. Khanna A, Gurusinghe D, Taylor D. Necrotizing myositis: highlighting the hidden depths - case series and review of the literature. ANZ J Surg. 2020;90:130-134.

18. George ME, Rueth NM, Skarda DE, Chipman JG, Quickel RR, Beilman GJ. Hyperbaric oxygen does not improve outcome in patients with necrotizing soft tissue infection. Surg Infect (Larchmt). 2009;10:21-28. 